\title{
On the two weights problem for the Hilbert transform
}

Nets Hawk Katz and Cristina Pereyra

\section{Introduction.}

In this paper, we prove sufficient conditions on pairs of weights $(u, v)$ (scalar, matrix or operator valued) so that the Hilbert transform

$$
H f(x)=\text { p.v. } \int \frac{f(y)}{x-y} d y,
$$

is bounded from $L^{2}(u)$ to $L^{2}(v)$. When $u=v$ are scalar, the classical results were given in [HMW] and [CF]. Earlier, [HS] gave a characterization of these weights by complex methods which has been generalized by [CS1] and [CS2] to the case of unequal weights. However these complex-analytic results give conditions which as stated by [CS2] "are not susceptible of being verified in practice". What follows shall be all in the category of real analysis. Matrix results for equal weights have recently been given in [TV]. For $u$ and $v$ scalar weights, a different sufficient condition from ours was given in $[\mathrm{F}]$. More general conditions than ours for the scalar case have recently been given by [TVZ] using very different methods which do not seem to generalize to the operator valued case.

We shall consider only $(u, v)$ so that $u^{-1}, v \in L_{\text {loc }}^{1+\varepsilon}$ are positive and $u^{-1}$ and $v$ are doubling. There will be an auxiliary Hilbert space $\mathcal{H}$, with scalar product denoted by $\langle\cdot, \cdot\rangle_{\mathcal{H}}$. The weights $u$ and $v$ shall be operator valued and we define for $\mathcal{H}$ valued functions $f$,

$$
\|f\|_{L^{2}(u)}^{2}=\int\langle u(x) f(x), f(x)\rangle_{\mathcal{H}} d x .
$$


Then we shall prove the following theorem:

Theorem. If $(u, v)$ as above satisfy conditions $\mathrm{a}), \mathrm{b})$ and $\mathrm{c})$ then

$$
H: L^{2}(u) \longrightarrow L^{2}(v) \text {. }
$$

For a full description of conditions a), b) and c) in the scalar and operator cases see Section 3. We briefly describe the conditions here.

Condition a) will state that for certain Haar multipliers $M_{u}$ and $M_{v}$, the operators

$$
u^{-1 / 2} M_{u}^{-1 / 2} \quad \text { and } \quad v^{1 / 2} M_{v}^{-1 / 2}
$$

are bounded on $L^{2}(\mathbb{R}, \mathcal{H})$. Operators of this form were first studied in [P1]. They were first used to study boundedness of the Hilbert transform in [TV]. In Section 1, we describe sufficient conditions for their boundedness in the scalar case. The weakness of these conditions, and their relation to the classical $A_{p}$ conditions on weights make condition a) seem reasonable.

Condition b) is a sort of non-local $A_{2}$ condition for $\left(v^{1+\varepsilon}, u^{-(1+\varepsilon)}\right)$. Condition $\mathrm{c}$ ) is the boundedness of two weighted paraproducts. (In the operator case, part of condition c) is also a seemingly slightly stronger assumption -an inequality that in the scalar case automatically follows. We point out that in the setting of [TV], this inequality may be replaced by the reverse inequality to $A_{2}$, i.e. the inequality

$$
\left(\frac{1}{|I|} \int_{I} w^{-1}\right)^{1 / 2}\left(\frac{1}{|I|} \int_{I} w\right)\left(\frac{1}{|I|} \int_{I} w^{-1}\right)^{1 / 2} \geq C
$$

which in the scalar case simply follows from Hölder's inequality. The inequality is also true in the operator valued case. For information on operator inequalities see $[\mathrm{HP}]$ and the references cited therein.)

In the matricial case when $u=v$, conditions a), b) and c) are equivalent to the classical Muckenhoupt $A_{2}$ condition.

Our theorem should be thought of as a sort of $T(1)$ theorem (see [D]) for two weights. In particular, condition c) should be seen as the analogue of requiring that $T(1)$ and $T^{*}(1)$ are in BMO. In this way, our proof differs from that of [TV] in the case that the weights are equal. We use only the standard kernel properties of the Hilbert transform $H$, namely the decay of matrix coefficients $H_{I J}$ when $3 I \cap 3 J=\varnothing$ and 
the general decay of $H h_{I}$. Further, we prove our bounds using not the Senechkin-Vinogradov test (as in [TV]) but rather the two fundamental lemmas of linear algebra:

Lemma 0.1 (Cotlar). Let $T_{j}$ be operators on $\mathcal{H}$, a Hilbert space. Suppose that for any $j, k$, one has

$$
\left\|T_{i} T_{j}^{*}\right\|_{\mathcal{H} \rightarrow \mathcal{H}} \leq a(|i-j|)
$$

and

$$
\left\|T_{j}^{*} T_{i}\right\|_{\mathcal{H} \rightarrow \mathcal{H}} \leq a(|i-j|),
$$

where $\sum_{j} a(j)^{1 / 2} \leq C$ then

$$
\left\|\sum_{j=-N}^{j=N} T_{j}\right\|_{\mathcal{H} \rightarrow \mathcal{H}} \leq C,
$$

with constant independent of $N$.

For a proof see [D]. Decomposition of an operator $T$ into $\sum_{j} T_{j}$ with the $T_{j}$ 's satisfying the hypotheses of the Lemma is called Cotlarization. The other fundamental lemma of linear algebra (in the scalar case), is

Lemma 0.2 (Schur). Let $T$ be an operator on $L^{2}(X)$ with $X$ a measure space and let $K(x, y)$, its scalar-valued kernel be positive. Suppose there are positive functions $w_{1}(x)$ and $w_{2}(x)$ with

$$
\int w_{1}(x) K(x, y) d x \leq C_{1} w_{2}(y)
$$

and

$$
\int w_{2}(y) K(x, y) d y \leq C_{2} w_{1}(x)
$$

Then $\|T\|_{L^{2}(X) \rightarrow L^{2}(X)} \leq\left(C_{1} C_{2}\right)^{1 / 2}$.

A proof may be found in [Da]. We state and prove a version in the operator case, (Lemma 3.1), which, while it is not deep, we have been unable to locate in the literature in this form.

Finally, we remark that the most important problem in the field of weighted norm inequalities for the Hilbert transform is to find the 
necessary and sufficient condition when $u=v$ in the case that $\mathcal{H}$ is not finite dimensional. It is conjectured that the condition is $A_{2}$. We do not know whether all $A_{2}$ weights satisfy our sufficient conditions, since the generalization of Gehring's theorem $[\mathrm{G}]$ is unclear. Also unclear is the correct definition for Carleson condition. We hope our paper inspires future work.

\section{Carleson conditions and bounded operators.}

We let $\mathcal{D}$ denote the set of dyadic intervals in the real line. We say that a sequence of real numbers $\left\{b_{I}\right\}$ indexed by $\mathcal{D}$ is a Carleson sequence provided that for any $I \in \mathcal{D}$, we have that

$$
\sum_{J \in \mathcal{D}, J \subset I} b_{J}^{2} \leq C|I|
$$

We recall the Carleson Lemma. (For a proof see [M, p. 261]).

Lemma 1.1 (Carleson). Let $\lambda_{I}$ be any sequence of real numbers. Define the function

$$
\lambda^{*}(x)=\sup _{x \in I}\left|\lambda_{I}\right|
$$

Then

$$
\left|\sum_{I} \lambda_{I} b_{I}^{2}\right| \leq C \int \lambda^{*}(x) d x
$$

For any interval $I$, we define $h_{I}$ to be the Haar function of $I$,

$$
h_{I}(x)=\frac{1}{|I|^{1 / 2}}\left(\chi_{I^{l}}-\chi_{I^{r}}\right)
$$

where $I^{l}$ and $I^{r}$ are the left and right children of $I$, the function $\chi_{J}$ for any interval $J$ is the characteristic function of $J$, and $|I|$ denotes the length of $I$. The $h_{I}$ 's form an orthonormal basis of $L^{2}(\mathbb{R})$. To any sequence $b_{I}$, we associate an operator $\pi_{b}$, its paraproduct by

$$
\pi_{b} f=\sum_{I \in \mathcal{D}} b_{I} h_{I} m_{I}(f)
$$

where $m_{I}(f)=\int_{I} f /|I|$ is the mean of $f$ on $I$. (More commonly, $\pi_{b}$ is referred to as the paraproduct with or by the BMO function $b=$ 
$\sum_{I \in \mathcal{D}} b_{I} h_{I}$. However throughout this paper the sequences $\left\{b_{I}\right\}$ occur far more naturally than the function $b$ and we prefer to think of $\pi_{b}$ as an operator associated to the sequence rather than as a modified product with the function).

Corollary 1.2. The operator $\pi_{b}$ is bounded on $L^{2}(\mathbb{R})$ if and only if $b$ is a Carleson sequence.

Proof. For one direction, we simply compute that if $b$ is a Carleson sequence,

$$
\left\|\pi_{b} f\right\|_{L^{2}(\mathbb{R})}^{2}=\sum_{I} b_{I}^{2}\left(m_{I} f\right)^{2} \leq C \int(\mathcal{M} f)^{2} \leq C\|f\|_{L^{2}(\mathbb{R})}^{2} .
$$

The first inequality follows from Lemma 1.1, where $\mathcal{M} f$ denotes the dyadic maximal function of $f$. The second inequality follows from the $L^{2}(\mathbb{R})$ boundedness of the dyadic maximal function, see $[\mathrm{D}]$. On the other hand, if $\pi_{b}$ is bounded then $\left\|\pi_{b} \chi_{I}\right\|_{L^{2}(\mathbb{R})}^{2} \leq C|I|$. However

$$
\left\|\pi_{b} \chi_{I}\right\|_{L^{2}(\mathbb{R})}^{2} \geq \sum_{J \subset I} b_{J}^{2}
$$

Hence $b$ is a Carleson sequence.

Throughout this section, $v$ shall be a weight -that is- a nonnegative $L_{\text {loc }}^{1}$ function, and $u_{I}$ and $b_{I}$ shall be sequences indexed by intervals (all intervals in the remainder of this paper shall be dyadic). We shall concern ourselves with two kinds of operators

$$
T_{v, u} f=v \sum_{I} \frac{\left\langle f, h_{I}\right\rangle}{u_{I}} h_{I}=v M_{u}^{-1} f
$$

and

$$
S_{v, u, b} f=M_{u}^{-1} \pi_{b}(v f)
$$

Here, obviously, $M_{u}$ denotes the Haar multiplier with coefficients $u_{I}$, and $\langle\cdot, \cdot\rangle$ denotes the scalar product in $L^{2}(\mathbb{R})$. In this section, following [TV], we shall show that the $L^{2}$ boundedness of the operators in (1.1) and (1.2) is related. We shall give sufficient conditions, and we 
shall demonstrate their relationship with the now classical CoifmanMuckenhoupt conditions on weights (see $[\mathrm{CF}]$ ).

It is clear that a necessary condition for $T_{v, u}$ to be bounded is that $m_{I}\left(v^{2}\right) \leq C u_{I}^{2}$. Let $b_{I}=\left\langle v^{2}, h_{I}\right\rangle / m_{I}\left(v^{2}\right)$. Then we have:

Proposition 1.3. If $m_{I}\left(v^{2}\right) \leq C u_{I}^{2}$ then $T_{v, u}$ is bounded on $L^{2}(\mathbb{R})$ if and only if $S_{v, u, b}$ is bounded on $L^{2}(\mathbb{R})$.

ProOF. First observe that since $m_{I}\left(v^{2}\right) \leq C u_{I}^{2}$, we have that

$$
S_{v, u, b}^{*} h_{I}=\frac{\left\langle v^{2}, h_{I}\right\rangle v \chi_{I}}{u_{I} m_{I}\left(v^{2}\right)|I|}
$$

is a bounded set in $L^{2}(\mathbb{R})$. Hence, $g_{I}=T_{v, u} h_{I}-S_{v, u, b}^{*} h_{I}$ is a bounded set in $L^{2}(\mathbb{R})$. If $g_{I}$ is also an orthogonal set in $L^{2}(\mathbb{R})$, then $T_{v, u}-S_{v, u, b}^{*}$ is a bounded operator on $L^{2}(\mathbb{R})$. Which would prove the proposition.

But in fact $g_{I}$ is an orthogonal set. To see this observe that for each interval $I$, the function $g_{I}$ is supported on the interval $I$ and that restricted to each of the left and right halves of $I$, it is a constant multiple of $v$. Thus to show that $g_{I}$ is an orthogonal set, it suffices to show that $g_{I} \perp v \chi_{I}$. But this is easy to verify since

$$
\int g_{I} v \chi_{I}=\frac{1}{u_{I}}\left(\int v^{2} h_{I}-\frac{\left\langle v^{2}, h_{I}\right\rangle}{|I| m_{I}\left(v^{2}\right)} \int_{I} v^{2}\right)=0
$$

which proves the proposition.

Next, we give a sufficient condition for $S_{v, u, b}$ to be bounded.

Proposition 1.4. Suppose there exists $\delta>0$ so that

$$
\left(\frac{\left(m_{I}\left(v^{2+\delta}\right)\right)^{1 /(2+\delta)}}{u_{I}}\right) b_{I}
$$

is a Carleson sequence. Then the operator $S_{v, u, b}$ is bounded on $L^{2}(\mathbb{R})$.

Proof. We have that

$$
\left\|S_{v, u, b} f\right\|_{L^{2}}^{2}=\sum_{I} \frac{b_{I}^{2}}{u_{I}^{2}}\left(m_{I}(v f)\right)^{2} .
$$


However by Hölder's inequality,

$$
m_{I}(v f) \leq\left(m_{I}\left(v^{2+\delta}\right)\right)^{1 /(2+\delta)}\left(m_{I}\left(f^{(2+\delta) /(1+\delta)}\right)\right)^{(1+\delta) /(2+\delta)} .
$$

Now simply applying Carleson's lemma and the boundedness of the dyadic maximal function on $L^{(2+2 \delta) /(2+\delta)}$ proves the proposition.

Corollary 1.5. Suppose $w \in \mathrm{RH}_{2}$, that is there exists a constant $C$ so that for any dyadic interval $I, m_{I}\left(w^{2}\right) \leq C\left(m_{I} w\right)^{2}$. Then $T_{w, m_{I}(w)}$ is bounded on $L^{2}(\mathbb{R})$.

Proof. If $w \in \mathrm{RH}_{2}$ then $w^{2} \in A_{\infty}$. Hence, $\left\langle w^{2}, h_{I}\right\rangle / m_{I}\left(w^{2}\right)=b_{I}$ is a Carleson sequence [FKP]. By Proposition 1.3, we need only show that $S_{w, m_{I} w, b}$ is bounded, but this follows immediately from the fact that for some $\delta \geq 0$, we have that $w \in \mathrm{RH}_{2+\delta}$ together with Proposition 1.4.

For other proofs, applications, and $L^{p}$ versions of Corollary 1.5, see [P1], [P2], [KP].

Corollary 1.6. Suppose that $w \in A_{2}$. Then the operators $T_{w^{1 / 2},\left(m_{I} w\right)^{1 / 2}}$ and $T_{w^{-1 / 2},\left(m_{I} w\right)^{-1 / 2}}$ are bounded on $L^{2}(\mathbb{R})$.

Proof. By propositions 1.3 and 1.4 , the operator $T_{w^{1 / 2},\left(m_{I} w\right)^{1 / 2}}$ is bounded for any $w \in A_{\infty}$. This follows from $\left\langle w, h_{I}\right\rangle / m_{I}(w)$ being a Carleson sequence, which occurs when $w \in A_{\infty}$, as well as the fact that $w \in \mathrm{RH}_{1+\varepsilon}$, for some $\varepsilon>0$. Now since $w^{-1} \in A_{\infty}$, we have that $T_{w^{-1 / 2},\left(m_{I}\left(w^{-1}\right)\right)^{1 / 2}}$ is bounded. But since $w \in A_{2}$, it is the case that $1 / m_{I}(w) \geq m_{I}\left(w^{-1}\right)$. This together with the boundedness of Haar multipliers with bounded coefficients proves the corollary.

For more information on the classical theory of Muckenhoupt weights, we refer the reader to [D].

We remark that Corollary 1.6 gives a trivial proof of the boundedness of Haar multipliers with bounded coefficients on $L^{2}(w)$ for any $w \in A_{2}$. The corollary says that $w^{1 / 2} M_{w}^{-1 / 2}$ and the adjoint of its inverse $w^{-1 / 2} M_{w}^{1 / 2}$ are bounded where $M_{w}$ is the Haar multiplier with coefficients $m_{I} w$. Let $L$ be a Haar multiplier with bounded coefficients. Then it is bounded on $L^{2}(w)$ if and only if $w^{1 / 2} L w^{-1 / 2}$ is bounded on $L^{2}(\mathbb{R})$. By the boundedness of the operators from Corollary 1.6 and their adjoints, this is true if and only if $M_{w}^{1 / 2} L M_{w}^{-1 / 2}$ is bounded on 
$L^{2}(\mathbb{R})$. But everything commutes and $M_{w}^{1 / 2} L M_{w}^{-1 / 2}=L$. Hence $L$ is bounded on $L^{2}(w)$.

Similarly, one has a simple proof that $\pi_{b}$ where $b$ is a Carleson sequence is bounded on $L^{2}(w)$ when $w \in A_{2}$. We simply observe that it suffices to show that $M_{w}^{1 / 2} \pi_{b} w^{-1 / 2}$ is bounded on $L^{2}(\mathbb{R})$. Now we apply Proposition 1.4 using the fact that $w \in A_{2}$ implies $w \in A_{2-\delta}$.

The same ideas can be used to give simple sufficient conditions for $L$ and $\pi_{b}$ to satisfy two weight inequalities. For example, $L$ takes $L^{2}(u) \longrightarrow L^{2}(v)$ provided there exist sequences $c_{1}$ and $c_{2}$ so that $T_{u^{-1 / 2}, c_{1}}$ and $T_{v^{1 / 2}, c_{2}}$ are bounded and $\left(c_{1}, c_{2}\right)$ satisfy an $A_{2}$ condition, i.e. $1 /\left(c_{1, I} c_{2, I}\right) \leq C$ for every dyadic $I$. Similarly, $\pi_{b}$ is bounded from $L^{2}(u)$ to $L^{2}(v)$ provided $T_{v^{1 / 2}, c_{2}}$ is bounded and there exists $\delta>0$ so that $\left(m_{I}\left(u^{-1-\delta / 2}\right)\right)^{1 /(2+\delta)} \leq C c_{2, I}$. The argument which proves this is the same as the proof of Proposition 1.4.

These ideas exactly form the basis for our two weights result for the Hilbert transform. Some pieces of the operator we will study will be treated like multipliers while others are treated like paraproducts. First, however, we discuss the relationship of the boundedness of $T_{v^{1 / 2}, c_{2}}$ to $v \in A_{\infty}$.

As mentioned in the proof of Corollary 1.6, for any $w \in A_{\infty}$, we have that $T_{w^{1 / 2},\left(m_{I}(w)\right)^{1 / 2}}$ is bounded. This followed from the fact that $w \in \mathrm{RH}^{1+\varepsilon}$, for some $\varepsilon>0$. In what follows, define for any $\varepsilon, w_{I, \varepsilon}=$ $\left(m_{I}\left(w^{1+\varepsilon}\right)\right)^{1 /(1+\varepsilon)}$. Fixing $\varepsilon>0$, we may ask when $T_{w^{1 / 2},\left(w_{I, \varepsilon}\right)^{1 / 2}}$ is bounded. Propositions 1.3 and 1.4 give as a sufficient condition that there exists a $\delta>0$ so that

$$
b_{I}=\frac{\left\langle w, h_{I}\right\rangle\left(w_{I, \delta}\right)^{1 / 2}}{\left(m_{I}(w)\right)\left(w_{I, \varepsilon}\right)^{1 / 2}}
$$

is a Carleson sequence. By Hölder's inequality, it is certainly sufficient that $c_{I}=\left\langle w, h_{I}\right\rangle / w_{I, \mu}$ is a Carleson sequence provided that $0<\mu<$ $\varepsilon /(2+\varepsilon)$. We do not necessarily get the result when $\mu=\varepsilon /(2+\varepsilon)$ since weights not in $A_{\infty}$ do not necessarily satisfy a reverse Hölder condition. When $c_{I}$ is a Carleson sequence we say that $w \in A_{\infty+\mu}$. Certainly, if $w \in A_{\infty}$ then $w \in A_{\infty+\mu}$. A priori, one might believe that any weight in $A_{\infty+\mu}$ is in $A_{\infty}$ or that all weights are in $A_{\infty+\mu}$. In fact, neither is the case. We thank Peter Jones for the following examples.

First consider $w(x)=\left|(\log x)^{-2} / x\right|$ on the interval [0,1/2]. We have that $w(x)$ is not in $A_{\infty}$ since it is not in $L^{1+\mu}$ for any $\mu$. However, on every interval not containing 0 , it satifies a reverse Hölder inequality 
with uniform estimates. Thus we have $w \in A_{\infty+\mu}$ since on those intervals $I$ containing 0 , we have $c_{I}=0$ while on the others we simply apply [FKP]. In other words, to sum $c_{I}^{2}$ for $I$ 's contained in an interval of the form $\left[0,2^{-j}\right]$, we need only sum it over intervals contained in intervals of the form $\left[2^{-k-1}, 2^{-k}\right]$ with $k>j$, apply [FKP] to each of these and sum the geometric series.

Next, we define a weight $w_{j}$ with the parameter $j$ an integer. The $A_{\infty+\mu}$ constant for any $\mu>0$ will be unbounded as we vary the parameter $j$. We define $f_{j,[0,1]}$ to be the function defined on the interval $[0,1]$ which takes on the value $2^{j}-\delta$ on $\left[0,2^{-j}\right]$, is constant on the rest of $[0,1]$ and has mean 1 . For any interval $I$, we let $f_{j, I}$ be the same function rescaled to the interval $I$. Let $w_{j, 0}=f_{j,[0,1]}$ We choose

$$
\delta \ll 1 /\left(2^{j}\right)^{\left(2^{j}\right)^{2^{j}}},
$$

so that we may neglect it for what follows. Now we define $w_{j, 1}$ by letting it equal $w_{j, 0}$ in the interval $\left[0,2^{-j}\right]$. Now in the interval $\left[k 2^{-j},(k+\right.$ $\left.1)\left(2^{-j}\right)\right]$ for $1 \leq k \leq 2^{j-1}$ we let $w_{j, 1}=w_{j, 0} f_{j,\left[k 2^{-j},(k+1)\left(2^{-j}\right)\right]}$. We repeat the procedure $2^{j}$ times letting $w_{j}=w_{j, 2^{j}}$. Now

$$
r_{j}=2^{j} \sum_{\left[0,2^{-j}\right] \subset J \subset[0,1]} \frac{\left\langle f_{j,[0,1]}, h_{J}\right\rangle^{2}}{f_{J}^{2}},
$$

can readily be seen to be comparable to the $A_{\infty+\varepsilon}$ constant of $w_{j}$ when

$$
f_{J}=\left(\frac{1}{|J|} \int_{J}\left(f_{j,[0,1]}\right)^{1+\varepsilon}\right)^{1 /(1+\varepsilon)} \text {. }
$$

But $r_{j}$ is readily seen to be approximately $2^{j /(1+\varepsilon)}$.

\section{A small section on operators.}

The purpose of this section is just to discuss the generalizations of Jensen's inequality and Schur's lemma which we shall be using in the proof of the main theorem.

From this point on, $\mathcal{H}$ will be a Hilbert space. We will think of $H$, the Hilbert transform as acting on $L^{2}(\mathbb{R}, \mathcal{H})$, the space of square integrable Hilbert space valued functions. This space is the same as $L^{2}(\mathbb{R}) \otimes \mathcal{H}$. Naturally, we define the action of $H$ by $H(f \otimes v)=(H f \otimes v)$. 
Our weights $u$ and $v$ shall be positive operator valued functions on $\mathcal{H}$. For any two self-adjoint operators $A$ and $B$, we say that $A \leq B$ when $B-A$ is positive; and for $C$ a constant, $A \leq C$ means that $(C \operatorname{Id}-A)$ is positive.

First, we state and prove the correct version of Schur's Lemma for operator valued kernels.

Lemma 2.1 (Schur). Let $X$ be a measure space. And let $K(x, y)$ be a $\mathcal{B}(\mathcal{H})$ valued function on $X \times X$. Suppose that $K(x, y)=A(x, y) B(x, y)$ where the multiplication is pointwise composition. Suppose further that

$$
\int A(x, y) A^{*}(x, y) d y \leq C_{1}
$$

and that

$$
\int B^{*}(x, y) B(x, y) d x \leq C_{2} \text {. }
$$

Then $K(x, y)$ gives rise to a bounded operator on $L^{2}(\mathbb{R}, \mathcal{H})$ with bound $C_{1}^{1 / 2} C_{2}^{1 / 2}$.

Proof. We need only to bound

$$
\int\langle f(x), K(x, y) g(y)\rangle_{\mathcal{H}} d x d y
$$

We observe

$$
\begin{aligned}
\int\langle f(x), & A(x, y) B(x, y) g(y)\rangle_{\mathcal{H}} d x d y \\
\leq & \int\left\langle A^{*}(x, y) f(x), B(x, y) g(y)\right\rangle_{\mathcal{H}} d x d y \\
\leq & \left(\int\left|A^{*}(x, y) f(x)\right|^{2} d x d y\right)^{1 / 2}\left(\int|B(x, y) g(y)|^{2} d x d y\right)^{1 / 2}
\end{aligned}
$$

here $|\cdot|$ denotes the norm in $\mathcal{H}$, i.e. $\|\cdot\|_{\mathcal{H}}=\left(\langle\cdot, \cdot\rangle_{\mathcal{H}}\right)^{1 / 2}$.

We write the first integral as

$$
\int\left\langle A(x, y) A^{*}(x, y) f(x), f(x)\right\rangle_{\mathcal{H}} d x d y
$$

and bound it by integrating first in $y$. We do the analogous thing for the second integral. 
Further we need to state the operator version of Jensen's inequality.

Lemma 2.2. Let $A(x)$ be a positive operator valued function on a measure space $X$. Let $d \mu(x)$ be a measure on $X$ with total measure 1 . Let $1 \leq p \leq \infty$. Then

$$
\left(\int A(x)^{p} d \mu(x)\right)^{1 / p} \geq \int A(x) d \mu(x) .
$$

For $1 \leq p \leq 2$, the only case in which we will use this, the result follows from [HP] and from the monotonicity of the function $f(t)=t^{r}$ when $0 \leq r \leq 1$, see [KR, Exercise 4.6.46]. All solutions are provided in [KR2]. Of course, we get immediately by scaling a version of Hölder's inequality:

Lemma 2.3. Let $A(x)$ be a positive operator valued function and let $f(x)$ be a scalar, positive, integrable function. Then

$$
\int f(x) A(x) d x \leq\left(\int f(x)\right)^{1 / q}\left(\int f(x) A(x)^{p} d x\right)^{1 / p}
$$

whenever $1<p<\infty$ and $1 / p+1 / q=1$.

ProOF. Simply apply Lemma 2.2 to the measure

$$
d \mu(x)=\frac{f(x) d x}{\int f(y) d y} .
$$

Many norm estimates will be based on

Lemma 2.4. Let $T_{1}$ and $T_{2}$ be positive operators with $T_{1} \leq T_{2}$. Let $S$ be any fixed operator. Then

$$
\left\|T_{1}^{1 / 2} S\right\| \leq\left\|T_{2}^{1 / 2} S\right\|
$$

Here $\|\cdot\|$ denotes the operator norm.

This is [KR, exercise 4.6.1]. 


\section{The two weights problem.}

In this section we will give a sufficient condition on pairs of doubling weights $(u, v)$ ensuring that the operator $v^{1 / 2} H u^{-1 / 2}$ is bounded where $H$ is the Hilbert transform. Here an operator valued weight $v$ is said to be doubling if there exists a constant $C$ so that for any dyadic interval $I$, whenever $\tilde{I}$ is its parent, one has

$$
\int_{\tilde{I}} v \leq C \int_{I} v
$$

with the inequality in the sense of operators.

As always $\mathcal{D}$ shall denote the set of all dyadic intervals in the real line. The set of dyadic intervals of length $2^{-k}$ shall be $\mathcal{D}_{k}$. We shall divide the set of all ordered pairs of dyadic intervals into a union of 5 disjoint sets. Let

$$
\begin{aligned}
& Z_{1}=\{(I, J):|I|>|J|, 3 I \cap 3 J=\varnothing\}, \\
& Z_{2}=\{(I, J):|I|<|J|, 3 I \cap 3 J=\varnothing\}, \\
& Z_{3}=\{(I, J):|I|<|J|, 3 I \cap 3 J \neq \varnothing\}, \\
& Z_{4}=\{(I, J):|I|>|J|, 3 I \cap 3 J \neq \varnothing\},
\end{aligned}
$$

and

$$
Z_{5}=\{(I, J):|I|=|J|\},
$$

We let $E_{I}$ denote the projection onto the Haar function on $I$, which we denote $h_{I}$. In other words, $E_{I} f=\left\langle f, h_{I}\right\rangle h_{I}$ for all $f \in L^{2}(\mathbb{R}, \mathcal{H})$, here $\left\langle f, h_{I}\right\rangle=\int f(x) h_{I}(x) d x \in \mathcal{H}$. We shall break up the Hilbert transform into corresponding pieces

$$
H_{\alpha}=\sum_{(I, J) \in Z_{\alpha}} E_{J} H E_{I} .
$$

Here $\alpha$ runs from 1 to 5 .

We now state our conditions on the pair $(u, v)$ and derive a few easy consequences.

First define

$$
u_{I}=\left(\frac{1}{|I|} \int u^{-(1+\varepsilon)}\right)^{1 /(1+\varepsilon)}
$$

and

$$
v_{I}=\left(\frac{1}{|I|} \int v^{1+\varepsilon}\right)^{1 /(1+\varepsilon)} .
$$


Here the number $\varepsilon>0$ shall be fixed throughout. (By Lemma 2.2, it is clear that if we define the mean $m_{I}(u)=\int_{I} u /|I|$ then one has the operator inequalities $m_{I}\left(u^{-1}\right) \leq u_{I}$ and $m_{I}(v) \leq v_{I}$ which we shall use frequently).

We define the operators acting on $L^{2}(\mathbb{R}, \mathcal{H})$

$$
T_{u} f=u^{-1 / 2} M_{u}^{-1 / 2} f=\sum_{I \in \mathcal{D}} u^{-1 / 2} u_{I}^{-1 / 2}\left\langle f, h_{I}\right\rangle h_{I}
$$

and

$$
T_{v} f=v^{1 / 2} M_{v}^{-1 / 2} f=\sum_{I \in \mathcal{D}} v^{1 / 2} v_{I}^{-1 / 2}\left\langle f, h_{I}\right\rangle h_{I} .
$$

Here $M_{w}^{t}$ denotes the Haar multiplier acting on $L^{2}(\mathbb{R}, \mathcal{H})$ with coefficient $w_{I}^{t}$, for $w_{I}$ a given sequence of positive selfadjoint operators on $\mathcal{H}$.

We say that $(u, v)$ satisfies condition a), provided that $T_{u}$ and $T_{v}$ are bounded operators on $L^{2}(\mathbb{R}, \mathcal{H})$.

We say that $(u, v)$ satisfy condition b) provided that

$$
u_{I}^{1 / 2}\left(\left(|I| \int_{(3 I)^{c}} \frac{v^{1+\varepsilon}}{\left(x-y_{I}\right)^{2}}\right)^{1 /(1+\varepsilon)}+v_{I}+v_{I-|I|}+v_{I+|I|}\right) u_{I}^{1 / 2} \leq C
$$

and that

$$
\begin{array}{r}
v_{I}^{1 / 2}\left(\left(|I| \int_{(3 I)^{c}} \frac{u^{-(1+\varepsilon)}}{\left(x-y_{I}\right)^{2}}\right)^{1 /(1+\varepsilon)}+u_{I}+u_{I-|I|}+u_{I+|I|}\right) v_{I}^{1 / 2} \\
\leq C
\end{array}
$$

where $y_{I}$ denotes the center of $I$. We observe that for any $A$ and $B$ positive operators, writing $B^{1 / 2} A B^{1 / 2} \leq C$ with $C$ a constant is the same as writing $\left\|A^{1 / 2} B^{1 / 2}\right\| \leq C^{1 / 2}$. We also point out that for any positive operator-valued function $w$, we have that

$$
\int_{(3 I)^{c}} \frac{w}{\left|x-y_{I}\right|^{n}} \leq \frac{1}{|I|^{n-2}} \int_{(3 I)^{c}} \frac{w}{\left|x-y_{I}\right|^{2}}, \quad \text { for } n>2 .
$$

This is not really Hölder's inequality but just the statement that on $(3 I)^{c}$, we have

$$
\frac{1}{\left|x-y_{I}\right|^{n-2}} \leq C \frac{1}{|I|^{n-2}} \text {. }
$$


Finally, we come to condition c). If $c_{I}$ is a sequence of bounded operators in $\mathcal{H}$ (not necessarilly selfadjoint this time) indexed by the dyadic intervals, we define the paraproduct

$$
\pi_{c} f=\sum_{I} h_{I} c_{I} m_{I}(f)
$$

Let $c_{I}^{v}$ be the operator on $\mathcal{H}$ given by $\left(m_{I}(v)\right)^{-1}\left\langle v, H_{3} h_{I}\right\rangle$ and $c_{I}^{u}$ the analogous thing for $u^{-1}$, where we define

$$
\left\langle v, H_{3} h_{I}\right\rangle=\int v(x)\left(H_{3} h_{I}\right)(x) d x .
$$

Then we say that $(u, v)$ satisfy condition c) provided that $M_{u}^{1 / 2} \pi_{c^{v}} v^{1 / 2}$ and $M_{v}^{1 / 2} \pi_{c^{u}} u^{-1 / 2}$ are bounded and that the following inequalities are satisfied for any dyadic $J \subset I$

$$
\begin{array}{r}
\left(m_{I}(v)\right)^{-1 / 2}\left(\frac{1}{|I|} \int_{I^{c}} v H_{3} h_{J}\right) u_{J}\left(\frac{1}{|I|} \int_{I^{c}} v H_{3} h_{J}\right)\left(m_{I}(v)\right)^{-1 / 2} \\
\leq C\left(\frac{|J|^{3}}{\left(d_{I J}\right)^{4}}\right) v_{I}^{-1 / 2} v_{J} v_{I}^{-1 / 2}
\end{array}
$$

and

$$
\begin{aligned}
&\left(m_{I}\left(u^{-1}\right)\right)^{-1 / 2}\left(\frac{1}{|I|} \int_{I^{c}} u^{-1} H_{3} h_{J}\right) v_{J} \\
& \cdot\left(\frac{1}{|I|} \int_{I^{c}} u^{-1} H_{3} h_{J}\right)\left(m_{I}\left(u^{-1}\right)\right)^{-1 / 2} \\
& \leq C\left(\frac{|J|^{3}}{\left(d_{I J}\right)^{4}}\right) u_{I}^{-1 / 2} u_{J} u_{I}^{-1 / 2} .
\end{aligned}
$$

Here $d_{I J}$ denotes the distance from $J$ to the boundary of $I$ and the inequalities are in the sense of operators. (By contrast, we will define $\rho_{I J}$ to be the maximum of $|I|,|J|$, and the distance between $I$ and $J$ ).

Let us observe a quick consequence of condition c). Let $h \in \mathcal{H}$ be a fixed vector. We apply the operator $M_{u}^{1 / 2} \pi_{c^{v}} v^{1 / 2}$ to the test function $v^{1 / 2} h \chi_{I}$. From the $I$-th summand of this we obtain the size estimate

$$
\left\|u_{I}^{1 / 2} c_{I}^{v}\left(m_{I}(v)\right)^{1 / 2}\right\|_{\mathcal{H} \rightarrow \mathcal{H}} \leq C|I|^{1 / 2} .
$$


There is of course an analogous inequality when the $u$ 's and $v$ 's are switched. In the case in which $u$ and $v$ are scalars, the inequality (3.5) (together with condition b)) implies the inequality (3.3).

We now give the proof of this implication. In the case where $v$ and $u$ are scalar, the definition of $c_{I}^{v}$ together with (3.5) implies that

$$
\int v H_{3} h_{I} \leq C \frac{|I|^{1 / 2}\left(m_{I}(v)\right)^{1 / 2}}{u_{I}^{1 / 2}}
$$

Now observe that $H_{3} h_{I}$ is constant on intervals whose length is $|I|$. Hence, $\left|H_{3} h_{I}\right| \leq C /|I|^{1 / 2}$ everywhere and is constant on $I$. Thus from (3.6),

$$
\int_{I^{c}} v H_{3} h_{I} \leq C\left(|I|^{1 / 2} m_{I}(v)+\frac{|I|^{1 / 2}\left(m_{I}(v)\right)^{1 / 2}}{u_{I}^{1 / 2}}\right) .
$$

But from condition $\mathrm{b}$ ) and the fact that $m_{I}(v) \leq v_{I}$, one has that

$$
\left(m_{I}(v)\right)^{1 / 2} \leq C \frac{1}{u_{I}^{1 / 2}}\left(m_{I}(v) u_{I}\right)^{1 / 2} \leq C \frac{1}{u_{I}^{1 / 2}}
$$

So that (3.7) implies that

$$
\int_{I^{c}} v H_{3} h_{I} \leq C \frac{|I|^{1 / 2}\left(m_{I}(v)\right)^{1 / 2}}{u_{I}^{1 / 2}} .
$$

Now we observe that on $I^{c}$, the function $H_{3} h_{I}$ is always positive. This is because $H h_{I}$ is positive on $I^{c}$ and $H_{3} h_{I}$ is given by the mean of $H h_{I}$ on a certain Whitney decomposition of $I^{c}$ (we will say more about this in the proof of Theorem 3.1). In fact, we have on $I^{c}$ that

$$
H_{3} h_{I}(x) \sim \frac{|I|^{3 / 2}}{\rho_{x I}^{2}}
$$

Where we define $\rho_{x I}$ to be the maximum of $|I|$ and the distance from $I$ to $x$. Thus one has

$$
\int_{I^{c}} v H_{3} h_{J} \leq C \frac{|J|^{3 / 2}|I|^{1 / 2}}{d_{I J}^{2}} \int_{I^{c}} v H_{3} h_{I},
$$


which immediately implies (3.3). In the last estimate we used the following facts,

$$
\int \frac{d x}{\rho_{x I}^{s}} \sim \frac{1}{|I|^{s-1}}
$$

for any $1<s$,

$$
\int_{I^{c}} \frac{d x}{\rho_{x J}^{2}} \sim \frac{1}{d_{I J}}
$$

for $J \subset I$.

This type of integral/series will appear repeatedly. Variations will be introduced as subtler sets/intervals are defined.

Theorem 3.1. Suppose $(u, v)$ satisfy conditions a), b) and c). Then the Hilbert transform $H$ is bounded from $L^{2}(u)$ to $L^{2}(v)$.

Proof. Our goal is to show that the operator $v^{1 / 2} H u^{-1 / 2}$ is bounded on $L^{2}(\mathbb{R}) \otimes \mathcal{H}$. By condition a) which states that operators $v^{1 / 2} M_{v}^{-1 / 2}$ and $u^{-1 / 2} M_{u}^{-1 / 2}$ are bounded, it would suffice to show that $M_{v}^{1 / 2} H M_{u}^{1 / 2}$ is bounded. In fact, we will show that $M_{v}^{1 / 2}\left(H_{1}+H_{2}+H_{5}\right) M_{u}^{1 / 2}$, as well as $v^{1 / 2} H_{3} M_{u}^{1 / 2}$ and $M_{v}^{1 / 2} H_{4} u^{-1 / 2}$ are bounded. Then we shall write

$$
\begin{aligned}
v^{1 / 2} H u^{-1 / 2}= & T_{v} M_{v}^{1 / 2}\left(H_{1}+H_{2}+H_{5}\right) M_{u}^{1 / 2} T_{u}^{*} \\
& +\left(v^{1 / 2} H_{3} M_{u}^{1 / 2}\right) T_{u}^{*}+T_{v}\left(M_{v}^{1 / 2} H_{4} u^{-1 / 2}\right),
\end{aligned}
$$

thereby proving the theorem.

By the symmetry between $u$ and $v$, proving that $M_{v}^{1 / 2} H_{1} M_{u}^{1 / 2}$ and $v^{1 / 2} H_{3} M_{u}^{1 / 2}$ are bounded is the same as proving that $M_{v}^{1 / 2} H_{2} M_{u}^{1 / 2}$ and $M_{v}^{1 / 2} H_{4} u^{-1 / 2}$ are bounded. The proof bounding $M_{v}^{1 / 2} H_{5} M_{u}^{1 / 2}$ is also exactly the same as the proof that $M_{v}^{1 / 2} H_{1} M_{u}^{1 / 2}$ is bounded once one makes the trivial observation that for any two intervals $I$ and $J$ of the same length, one has

$$
\left|H_{I J}\right| \leq C \frac{|I|^{3 / 2}|J|^{3 / 2}}{\rho_{I J}^{3}},
$$


where $\rho_{I J}$ is the maximum of $|I|,|J|$, and the distance between $I$ and $J$ and where $H_{I J}=\left\langle H h_{I}, h_{J}\right\rangle$.

Thus we shall proceed to prove only that the operators $M_{v}^{1 / 2} H_{1}$ - $M_{u}^{1 / 2}$ and $v^{1 / 2} H_{3} M_{u}^{1 / 2}$ are bounded on $L^{2}(\mathcal{H})$.

We begin with $M_{v}^{1 / 2} H_{1} M_{u}^{1 / 2}$. We shall denote its matrix coefficients by $K_{I J}$. Each is a linear operator on $\mathcal{H}$. We have that for $|I|>|J|$ with $3 I \cap 3 J=\varnothing$,

$$
K_{I J}=u_{I}^{1 / 2} H_{I J} v_{J}^{1 / 2}
$$

For these $(I, J)$ 's, one has the classical estimate see [Da,TV],

$$
\left|H_{I J}\right| \leq C \frac{|I|^{3 / 2}|J|^{3 / 2}}{\rho_{I J}^{3}}
$$

Throughout this section whenever $A$ is a real scalar or more generally a self-adjoint operator, we shall, by abuse of notation denote by $A^{1 / 2}$ some choice of normal square root for $A$ always using the fact that $A^{1 / 2}\left(A^{1 / 2}\right)^{*}=|A|$ where $|A|$ denotes the sum of the positive and negative parts of $A$.

We apply Lemma 2.1 to $K_{I J}$. We let $A_{I J}=u_{I}^{1 / 2} v_{J}^{1 / 2} H_{I J}^{1 / 2}$. Hence, we let $B_{I J}=H_{I J}^{1 / 2}$. The desired estimate on $\sum_{I} B_{I J}^{*} B_{I J}$ is simply the corresponding estimate for the scalar Hilbert transform. We need only bound

$$
\sum_{\substack{J:|J|<|I| \\ 3 J \cap 3 I=\varnothing}} u_{I}^{1 / 2} v_{J}\left|H_{I J}\right| u_{I}^{1 / 2} \leq C u_{I}^{1 / 2}\left(\sum_{\substack{J:|J|<|I| \\ 3 J \cap 3 I=\varnothing}} \frac{v_{J}|J|^{3 / 2}|I|^{3 / 2}}{\rho_{I J}^{3}}\right) u_{I}^{1 / 2}
$$

where the last inequality is in the sense of positive operators. Suppose that $I \in \mathcal{D}_{j}$. Then we subdivide into a sum over the intervals $J \in \mathcal{D}_{k}$ and over all $k>j$. Hence,

$$
\sum_{J \in \mathcal{D}_{k}} A_{I J} A_{I J}^{*} \leq C \sum_{k>j} u_{I}^{1 / 2}\left(\sum_{\substack{J: J \in \mathcal{D}_{k} \\ 3 J \cap 3 I=\varnothing}} \frac{v_{J}|J|^{3 / 2}|I|^{3 / 2}}{\rho_{I J}^{3}}\right) u_{I}^{1 / 2}
$$


Now we estimate

$$
\begin{aligned}
& \sum_{\substack{J: J \in \mathcal{D}_{k} \\
3 J \cap 3 I=\varnothing}} \frac{v_{J}|J|^{3 / 2}|I|^{3 / 2}}{\rho_{I J}^{3}} \\
& \leq\left(\sum_{J \in \mathcal{D}_{k}} \frac{|I|^{3 / 2}|J|^{3 / 2}}{\rho_{I J}^{3}}\right)^{\varepsilon /(1+\varepsilon)} \\
& \cdot\left(\sum_{\substack{J \in \mathcal{D}_{k} \\
3 J \cap 3 I=\varnothing}} \frac{|I|^{3 / 2}|J|^{3 / 2} v_{J}^{1+\varepsilon}}{\rho_{I J}^{3}}\right)^{1 /(1+\varepsilon)} \\
& \leq C\left(\frac{|J|^{1 / 2}}{|I|^{1 / 2}}\right)^{\varepsilon /(1+\varepsilon)}\left(\sum_{\substack{J \in \mathcal{D}_{k} \\
3 J \cap 3 I=\varnothing}} \frac{|J|^{3 / 2}|I|^{1 / 2} v_{J}^{1+\varepsilon}}{\rho_{I J}^{2}}\right)^{1 /(1+\varepsilon)} \\
& \leq C \frac{|J|^{1 / 2}}{|I|^{1 / 2}}\left(|I| \int_{(3 I)^{c}} \frac{v^{1+\varepsilon}}{\left(x-y_{I}\right)^{2}}\right)^{1 /(1+\varepsilon)} \cdot
\end{aligned}
$$

Now plugging (3.9) into (3.8) and using condition b) we conclude

$$
\sum_{J} A_{I J} A_{I J}^{*} \leq \sum_{k>j} \frac{|J|^{1 / 2}}{|I|^{1 / 2}} .
$$

But this is a geometric sum, so that $M_{v}^{1 / 2} H_{1} M_{u}^{1 / 2}$ is bounded.

For the penultimate inequality in (3.9) we used the fact that

$$
\sum_{J \in D_{k}} \frac{1}{\rho_{I J}^{s}} \sim \frac{|I|}{|J||I|^{s}},
$$

for $1<s,|J|<|I|$; which we can compare to

$$
\sum_{I \in D_{j}} \frac{1}{\rho_{I J}^{s}} \sim \frac{1}{|I|^{s}} \sim \sum_{I \in D_{j}} \frac{1}{\rho_{x I}^{s}}
$$

for $1<s,|J|<|I|$, and $x \in J$.

Now, we write $L=v^{1 / 2}\left(H_{3}-\pi_{c^{v}}^{*}\right) M_{u}^{-1 / 2}$. If we can bound the operator $L$ then we have proven the theorem by condition c). We shall apply Cotlar's lemma writing $L=\sum_{j} L_{j}$ with $L_{j}=L \Delta_{j}$, where $\Delta_{j}=\sum_{I \in \mathcal{D}_{j}} E_{I}$. We must bound $L_{k}^{*} L_{j}$, and it will be enough to 
consider only $k \leq j$ by symmetry considerations the case $k \geq j$ can be done using the half of hypothesis a), b), c) that are not used in what follows. Also we should not worry about bounding $L_{k} L_{j}^{*}$ because it can be seen that for $k \neq j$ one has $L_{k} L_{j}^{*}=0$.

We write with $I \in \mathcal{D}_{j}, J \in \mathcal{D}_{k}$ and $z_{1}, z_{2} \in \mathcal{H}$ that

$$
\left\langle L\left(h_{I} \otimes z_{1}\right), L\left(h_{J} \otimes z_{2}\right)\right\rangle_{L^{2}(\mathbb{R}, \mathcal{H})}=\sum_{\alpha=1}^{6}\left\langle z_{1}, L_{I J}^{\alpha} z_{2}\right\rangle_{\mathcal{H}}
$$

To define the decomposition $L^{\alpha}$ we now define a set of intervals $B_{j k}$. An interval $J$ is contained in $B_{j k}$ precisely if $J \in \mathcal{D}_{k}$ and there exists a $I \in \mathcal{D}_{j}$ so that the distance between $J$ and $\partial I$ is bounded by $|I|^{1 / 2}|J|^{1 / 2}$. Now we define

$$
\left\langle z_{1}, L_{I J}^{1} z_{2}\right\rangle_{\mathcal{H}}=\left\langle v^{1 / 2} H_{3} h_{I} u_{I}^{1 / 2} z_{1}, v^{1 / 2} H_{3} h_{J} u_{J}^{1 / 2} z_{2}\right\rangle_{L^{2}(\mathbb{R}, \mathcal{H})},
$$

when $J \in B_{j k}$ and 0 otherwise,

$$
-\left\langle z_{1}, L_{I J}^{2} z_{2}\right\rangle_{\mathcal{H}}=\left\langle v^{1 / 2} H_{3} h_{I} u_{I}^{1 / 2} z_{1}, v^{1 / 2} \pi_{c^{v}}^{*} M_{u}^{1 / 2}\left(h_{J} \otimes z_{2}\right)\right\rangle_{L^{2}(\mathbb{R}, \mathcal{H})},
$$

when $J \in B_{j k}$ and 0 otherwise,

$$
-\left\langle z_{1}, L_{I J}^{3} z_{2}\right\rangle_{\mathcal{H}}=\left\langle v^{1 / 2} \pi_{c^{v}}^{*} M_{u}^{1 / 2}\left(h_{I} \otimes z_{1}\right), v^{1 / 2} H_{3} h_{J} u_{J}^{1 / 2} z_{2}\right\rangle_{L^{2}(\mathbb{R}, \mathcal{H})},
$$

when $J \in B_{j k}$ and 0 otherwise,

$$
\left\langle z_{1}, L_{I J}^{4} z_{2}\right\rangle_{\mathcal{H}}=\left\langle v^{1 / 2} \pi_{c^{v}}^{*} M_{u}^{1 / 2}\left(h_{I} \otimes z_{1}\right), v^{1 / 2} \pi_{c^{v}}^{*} M_{u}^{1 / 2}\left(h_{J} \otimes z_{2}\right)\right\rangle_{L^{2}(\mathbb{R}, \mathcal{H})}
$$

when $J \in B_{j k}$ and 0 otherwise,

$$
\left\langle z_{1}, L_{I J}^{5} z_{2}\right\rangle_{\mathcal{H}}=\left\langle v^{1 / 2} H_{3} h_{I} u_{I}^{1 / 2} z_{1}, L\left(h_{J} \otimes z_{2}\right)\right\rangle_{L^{2}(\mathbb{R}, \mathcal{H})}
$$

when $J \notin B_{j k}$ and 0 otherwise and

$$
-\left\langle z_{1}, L_{I J}^{6} z_{2}\right\rangle_{\mathcal{H}}=\left\langle v^{1 / 2} \pi_{c^{v}}^{*} M_{u}^{1 / 2}\left(h_{I} \otimes z_{1}\right), L\left(h_{J} \otimes z_{2}\right)\right\rangle_{L^{2}(\mathbb{R}, \mathcal{H})},
$$

when $J \notin B_{j k}$ and 0 otherwise.

It suffices to bound the operator-valued matrices $L^{\alpha}$ with exponential decay in $|j-k|$, and this is what we shall do.

The main point of the argument is as follows. By the definition of $H_{3}$, the Haar expansion of $H_{3} h_{I}$ is the sum of all components 
$\left\langle H h_{I}, h_{J}\right\rangle h_{J}$ for $J$ such that $3 I \cap 3 J \neq \varnothing$ and $|I|<|J|$, denote that collection of intervals by $Z_{3}(I)$, for each $I$. The dyadic intervals $J$ with the property $3 I \cap 3 J=\varnothing$ which have $|I| \geq|J|$ and such that their parents $\tilde{J}$ belong to $Z_{3}(I)$ form a disjoint covering of $(5 I)^{c}$ and we may define $J_{I}(x)$ for any point $x$ in $(5 I)^{c}$ to be the element of this covering containing $x$. For $x \in 5 I$ we define $J_{I}(x)$ to be the dyadic interval of length $|I|$ containing $x$.

Then, by definition, we have that

$$
\left(H_{3} h_{I}\right)(x)=m_{J_{I}(x)}\left(H h_{I}\right) .
$$

Thus while $H h_{I}$ has logarithmic singularities, the function $H_{3} h_{I}$ does not. In fact, we have the precise size estimates which we shall use from now on

$$
\left|H_{3} h_{I}(x)\right| \leq C \frac{|I|^{3 / 2}}{\rho_{x I}^{2}} .
$$

Let us state some facts that will be used often in the proof. We let $S_{j k}=2\left(\cup_{J \in B_{j k}} J\right)$. For $I \in \mathcal{D}_{j}, J \in \mathcal{D}_{k}$, and $j<k$,

$$
\sum_{J \in B_{j k}} \frac{1}{\rho_{x J}^{2}} \leq \frac{C}{|J|^{2}},
$$

for $x \in S_{j k}$,

$$
\sum_{J \in B_{j k}} \frac{1}{\rho_{x J}^{2}} \leq \frac{C}{|J||I|}
$$

for $x \in S_{j k}^{c}$,

$$
\int_{S_{j k}} \frac{1}{\rho_{x I}^{2}} \leq C \frac{|J|^{1 / 2}}{|I|^{3 / 2}}
$$

We begin with

$$
L_{I J}^{1}=u_{I}^{1 / 2}\left(\int v\left(H_{3} h_{I}\right)\left(H_{3} h_{J}\right)\right) u_{J}^{1 / 2} .
$$

We apply Lemma 2.1, letting

$$
A_{I J}=u_{I}^{1 / 2}\left(\int v\left(H_{3} h_{I}\right)\left(H_{3} h_{J}\right)\right)^{1 / 2}
$$


and

$$
B_{I J}=\left(\int v\left(H_{3} h_{I}\right)\left(H_{3} h_{J}\right)\right)^{1 / 2} u_{J}^{1 / 2}
$$

We let $S_{j k}=2\left(\cup_{J \in B_{j k}} J\right)$ and shall estimate separately the integral on $S_{j k}$ and on $S_{j k}^{c}$. We must estimate

$$
\begin{aligned}
\sum_{J \in B_{j k}} A_{I J} A_{I J}^{*} & =\sum_{J \in B_{j k}} u_{I}^{1 / 2}\left|\int v\left(H_{3} h_{I}\right)\left(H_{3} h_{J}\right)\right| u_{I}^{1 / 2} \\
& \leq \sum_{J \in B_{j k}} u_{I}^{1 / 2}\left(\int_{S_{j k}} \frac{v|I|^{3 / 2}|J|^{3 / 2}}{\rho_{x I}^{2} \rho_{x J}^{2}}+\int_{S_{j k}^{c}} \frac{v|I|^{3 / 2}|J|^{3 / 2}}{\rho_{x I}^{2} \rho_{x J}^{2}}\right) u_{I}^{1 / 2}
\end{aligned}
$$

Here again $|\cdot|$ denotes the sum of the positive and negative parts. Now, we estimate the integrals using the trivial bound $|J| \leq \rho_{x J}$, Hölder, (v) and (vii),

$$
\begin{aligned}
\sum_{J \in B_{j k}} \int_{S_{j k}} \frac{v|I|^{3 / 2}|J|^{3 / 2}}{\rho_{x I}^{2} \rho_{x J}^{2}}= & \int_{S_{j k}} \frac{v|I|^{3 / 2}}{\rho_{x I}^{2}}\left(\sum_{J \in B_{j k}} \frac{|J|^{3 / 2}}{\rho_{x J}^{2}}\right) \\
\leq & \left(\frac{|I|}{|J|}\right)^{1 / 2}\left(|I| \int_{S_{j k}} \frac{v}{\rho_{x I}^{2}}\right) \\
\leq & C\left(\frac{|I|}{|J|}\right)^{1 / 2}\left(|I| \int_{S_{j k}} \frac{v^{1+\varepsilon}}{\rho_{x I}^{2}}\right)^{1 /(1+\varepsilon)} \\
& \cdot\left(|I| \int_{S_{j k}} \frac{1}{\rho_{x I}^{2}}\right)^{\varepsilon /(1+\varepsilon)} \\
\leq & C\left(\frac{|I|}{|J|}\right)^{1 / 2}\left(\frac{\left|S_{j k} \cap I\right|}{|I|}\right)^{\varepsilon /(1+\varepsilon)} \\
& \cdot\left(|I| \int \frac{v^{1+\varepsilon}}{\rho_{x I}^{2}}\right)^{1 /(1+\varepsilon)} \\
\leq & C\left(\frac{|I|}{|J|}\right)^{1 / 2-\varepsilon /(2+2 \varepsilon)}\left(|I| \int \frac{v^{1+\varepsilon}}{\rho_{x I}^{2}}\right)^{1 /(1+\varepsilon)} .
\end{aligned}
$$

Here the factor of $\left(\left|S_{j k} \cap I\right| /|I|\right)$ comes from the fact that $\rho_{x I}$ is almost constant on intervals of length $I$, also remember that $\left|S_{j k} \cap I\right| \sim$ $(|I||J|)^{1 / 2}$. However for $x \in S_{j k}^{c}$ we may use that $\rho_{x J} \geq(|I||J|)^{1 / 2}$, 
thus obtaining, using (vi), and Hölder,

$$
\begin{aligned}
\sum_{J \in B_{j k}} \int_{S_{j k}^{c}} \frac{v|I|^{3 / 2}|J|^{3 / 2}}{\rho_{x I}^{2} \rho_{x J}^{2}} \leq & \left(\frac{|J|}{|I|}\right)^{1 / 2}\left(|I| \int_{S_{j k}^{c}} \frac{v}{\rho_{x I}^{2}}\right) \\
\leq & C\left(\frac{|J|}{|I|}\right)^{1 / 2}\left(|I| \int \frac{v}{\rho_{x I}^{2}}\right) \\
\leq & C\left(\frac{|J|}{|I|}\right)^{1 / 2}\left(|I| \int \frac{1}{\rho_{x I}^{2}}\right)^{\varepsilon /(1+\varepsilon)} \\
& \cdot\left(|I| \int \frac{v^{1+\varepsilon}}{\rho_{x I}^{2}}\right)^{1 /(1+\varepsilon)} \\
\leq & C\left(\frac{|J|}{|I|}\right)^{1 / 2}\left(|I| \int \frac{v^{1+\varepsilon}}{\rho_{x I}^{2}}\right)^{1 /(1+\varepsilon)}
\end{aligned}
$$

Now we plug (3.12) and (3.13) into (3.11) using condition b) to obtain

$$
\sum_{J \in B_{j k}} A_{I J} A_{I J}^{*} \leq C\left(\left(\frac{|J|}{|I|}\right)^{1 / 2}+\left(\frac{|I|}{|J|}\right)^{1 / 2-\varepsilon /(2+2 \varepsilon)}\right) .
$$

We compute directly

$$
\sum_{I \in \mathcal{D}_{j}} B_{I J}^{*} B_{I J} \leq \sum_{I \in \mathcal{D}_{j}} u_{J}^{1 / 2}\left(\int \frac{v|I|^{3 / 2}|J|^{3 / 2}}{\rho_{x I}^{2} \rho_{x J}^{2}}\right) u_{J}^{1 / 2} \leq C \frac{|J|^{1 / 2}}{|I|^{1 / 2}}
$$

Here the last inequality follows from summing inside the integral, and then applying Hölder, condition b), (i) and (iv) as in (3.12) and (3.13). But this provides the desired estimates on $L_{I J}^{1}$ since the product of the estimates on $\sum_{J \in \mathcal{D}_{k}} A_{I J} A_{I J}^{*}$ and on $\sum_{I \in \mathcal{D}_{j}} B_{I J}^{*} B_{I J}$ decays exponentially in $k-j$.

We will use repeatedly the estimate deduced by Hölder and b) in $(3.13)$

$$
u_{I}^{1 / 2}\left(\int \frac{|I| v(x)}{\rho_{x I}^{2}}\right) u_{I}^{1 / 2} \leq C .
$$

To bound $L_{I J}^{2}$, naturally, we shall use condition c), recalling the estimate (3.5) namely

$$
\left\|u_{I}^{1 / 2} c_{I}^{v}\left(m_{I}(v)\right)^{1 / 2}\right\|_{\mathcal{H} \rightarrow \mathcal{H}} \leq C|I|^{1 / 2} .
$$


We abbreviate $D_{I}=u_{I}^{1 / 2} c_{I}^{v}\left(m_{I}(v)\right)^{1 / 2}$. By definition, we have that for $J \in B_{j k}$, and $x_{J} \in J$

$$
L_{I J}^{2}=u_{I}^{1 / 2}\left(H_{3} h_{I}\right)\left(x_{J}\right)\left(m_{J}(v)\right)^{1 / 2} D_{J}^{*}
$$

remember that

$$
\pi_{c^{v}}^{*} M_{u}^{1 / 2}\left(h_{J} \otimes z\right)=\frac{\chi_{J}(x)}{|J|}\left(c_{J}^{v}\right)^{*} u_{J}^{1 / 2} z
$$

We apply Lemma 3.1, letting

$$
A_{I J}=u_{I}^{1 / 2}\left(m_{J}(v)\right)^{1 / 2} \frac{|I|^{1 / 2}|J|^{1 / 2}}{\rho_{I J}}
$$

and letting

$$
B_{I J}=\frac{\rho_{I J}}{|I|^{1 / 2}|J|^{1 / 2}}\left(H_{3} h_{I}\right)\left(x_{J}\right) D_{J}^{*}
$$

Now we compute

$$
\begin{aligned}
\sum_{J \in B_{j k}} A_{I J} A_{I J}^{*} & =u_{I}^{1 / 2}|I|\left(\sum_{J \in B_{j k}} \frac{|J| m_{J} v}{\rho_{I J}^{2}}\right) u_{I}^{1 / 2} \\
& \leq C u_{I}^{1 / 2}\left(|I| \int_{S_{j k}} \frac{v}{\rho_{x I}^{2}}\right) u_{I}^{1 / 2} \\
& \leq C\left(\frac{|J|}{|I|}\right)^{\varepsilon /(2+2 \varepsilon)} u_{I}^{1 / 2}\left(|I| \int \frac{v^{1+\varepsilon}}{\rho_{x I}^{2}}\right)^{1 /(1+\varepsilon)} u_{I}^{1 / 2} \\
& \leq C\left(\frac{|J|}{|I|}\right)^{\varepsilon /(2+2 \varepsilon)} .
\end{aligned}
$$

Here $S_{j k}$ is as in (3.12), the penultimate inequality comes from the same application of Hölder as in (3.12), and the final inequality comes from condition b). On the other hand, by (3.5), we see that

$$
\sum_{I} B_{I J}^{*} B_{I J} \leq \sum_{I} \frac{|I|^{2}}{\rho_{I J}^{2}} \leq C .
$$

Hence, we have obtained the desired estimate for $L_{I J}^{2}$.

Next, we estimate $L_{I J}^{3}$. We obtain immediately from the definition that

$$
-L_{I J}^{3}=\frac{1}{|I|} u_{I}^{1 / 2} c_{I}^{v}\left(\int_{I} v\left(H_{3} h_{J}\right)\right) u_{J}^{1 / 2} .
$$


As before, we shall use that from condition c), we have (3.5). We apply Lemma 3.1, letting

$$
A_{I J}=\frac{|J|^{1 / 4}}{|I|} u_{I}^{1 / 2} c_{I}^{v}\left(\int_{I} v H_{3} h_{J}\right)^{1 / 2}
$$

and letting

$$
B_{I J}=|J|^{-1 / 4}\left(\int_{I} v H_{3} h_{J}\right)^{1 / 2} u_{J}^{1 / 2}
$$

We compute

$$
\sum_{J \in B_{j k}} A_{I J} A_{I J}^{*} \leq \sum_{J \in B_{j k}} \frac{1}{|I|^{2}} u_{I}^{1 / 2} c_{I}^{v}\left(\int_{I}|J|^{2} \frac{v}{\rho_{x J}^{2}}\right)\left(c_{I}^{v}\right)^{*} u_{I}^{1 / 2}
$$

by plugging in (3.14) into the sum and using the size estimates on $H_{3} h_{J}$. Now, we estimate the integral in (3.15) by breaking up the interval $I$ into $I \cap S_{j k}$ and $I \cap S_{j k}^{c}$ observing, by summing under the integral and using (v) and (vi), that

$$
\sum_{J \in B_{j k}} \int_{I}|J|^{2} \frac{v}{\rho_{x J}^{2}} \leq C\left(\int_{S_{j k} \cap I} v+\frac{|J|^{1 / 2}}{|I|^{1 / 2}} \int_{I} v\right) .
$$

The second piece in (3.16) is clearly bounded by $|J|^{1 / 2}|I|^{1 / 2} m_{I}(v)$. As for the first piece, we use doubling observing that $S \cap I$ is contained in the rightmost and leftmost dyadic subintervals of $I$ having measure more that $2|J|^{1 / 2}|I|^{1 / 2}$. Recall doubling implies that if $K$ is any dyadic interval and $\tilde{K}$ its parent then

$$
\int_{\tilde{K}} v \leq C \int_{K} v
$$

Now let $K_{b}$ be $K$ 's twin sister. Since $\int_{K} v \geq \int_{\tilde{K}} v / C$ while $\int_{\tilde{K}} v=$ $\int_{K} v+\int_{K_{b}} v$, one has that

$$
\int_{K_{b}} v \leq\left(1-\frac{1}{C}\right) \int_{\tilde{K}} v .
$$

Naturally, the same holds for $K$ by applying the doubling condition on $K_{b}$. In fact if $K^{\prime}$ is any descendant of $K$ after $l$ generations, one has

$$
\int_{K^{\prime}} v \leq\left(1-\frac{1}{C}\right)^{l} \int_{K} v \leq\left(\frac{\left|K^{\prime}\right|}{|K|}\right)^{\delta} \int_{K} v
$$


where $\delta \geq 0$ depends only on the doubling constant. Now since $S \cap I$ is contained in two descendants of $I$ of length at most $4|J|^{1 / 2}|I|^{1 / 2}$, one has that

$$
\int_{S \cap I} v \leq C\left(\frac{|J|}{|I|}\right)^{\delta / 2} \int_{I} v
$$

Plugging our observations into (3.16) yields that

$$
\sum_{J \in B_{j k}} \int_{I}|J|^{2} \frac{v}{\rho_{x J}^{2}} \leq C\left(|I|^{1-\delta / 2}|J|^{\delta / 2}+|I|^{1 / 2}|J|^{1 / 2}\right) m_{I}(v) .
$$

Now we plug (3.18) into (3.15), applying (3.5) and the fact that when $P_{1} \leq P_{2}$ then $T P_{1} T^{*} \leq T P_{2} T^{*}$ for any $P_{1}, P_{2}$, and $T$ to obtain that

$$
\sum_{J \in B_{j k}} A_{I J} A_{I J}^{*} \leq C\left(\left(\frac{|J|}{|I|}\right)^{\delta / 2}+\left(\frac{|J|}{|I|}\right)^{1 / 2}\right) .
$$

Now,

$$
\sum_{I \in \mathcal{D}_{j}} B_{I J}^{*} B_{I J} \leq \sum_{I \in \mathcal{D}_{j}} u_{J}^{1 / 2}\left(\int_{I} \frac{|J| v}{\rho_{x J}^{2}}\right) u_{J}^{1 / 2} \leq u_{J}^{1 / 2}\left(\int \frac{|J| v}{\rho_{x J}^{2}}\right) u_{J}^{1 / 2} \leq C .
$$

Here we have used Hölder and condition b) as in (3.12) and (3.13). Thus, we have obtained the desired estimates on $L_{I J}^{3}$.

We come now to $L_{I J}^{4}$. By definition, when $J \in B_{j k}$ and $J \subset I$,

$$
\begin{aligned}
L_{I J}^{4} & =u_{I}^{1 / 2} c_{I}^{v}\left(\int_{J} \frac{v}{|I||J|}\right)\left(c_{J}^{v}\right)^{*} u_{J}^{1 / 2} \\
& =\left(\frac{1}{|I|}\right) D_{I}\left(m_{I}(v)\right)^{-1 / 2}\left(m_{J}(v)\right)^{1 / 2} D_{J}^{*},
\end{aligned}
$$

when $J \cap I=\varnothing$ then by support considerations $L_{I J}^{4}=0$.

As usual we apply Lemma 2.1, though the sum over $I$ will be over a set with only one element. We let

$$
A_{I J}=\frac{|J|^{1 / 2}}{|I|} D_{I}\left(m_{I}(v)\right)^{-1 / 2}\left(m_{J}(v)\right)^{1 / 2}
$$

and

$$
B_{I J}=\frac{1}{|J|^{1 / 2}} D_{J}^{*}
$$


We compute

$$
\begin{aligned}
\sum_{\substack{J \in B_{j k} \\
J \subset I}} A_{I J} A_{I J}^{*} & =\sum_{\substack{J \in B_{j k} \\
J \subset I}}\left(\frac{1}{|I|}\right) D_{I}\left(\frac{|J|}{|I|}\right)\left(m_{I}(v)\right)^{-1 / 2}\left(m_{J}(v)\right)\left(m_{I}(v)\right)^{-1 / 2} D_{I}^{*} \\
& \leq\left(\frac{1}{|I|}\right)^{2} D_{I}\left(m_{I}(v)\right)^{-1 / 2} \int_{S \cap I} v\left(m_{I}(v)\right)^{-1 / 2} D_{I}^{*} \\
& \leq C\left(\frac{1}{|I|}\right) D_{I}\left(\frac{|J|}{|I|}\right)^{\delta / 2} D_{I}^{*} \\
& \leq C\left(\frac{|J|}{|I|}\right)^{\delta / 2}
\end{aligned}
$$

Here the penultimate estimate is by (3.17) and the last one by (3.5). The bound on $B_{I J}^{*} B_{I J}$ independent of $J$ is just (3.5). Hence, $L_{I J}^{4}$ satisfies the desired estimates.

Next we bound $L_{I J}^{5}$. This time $J \notin B_{j k}$. We define

$$
\hat{J}=\left(\frac{|I|^{1 / 2}}{|J|^{1 / 2}}\right) J
$$

and we have that if $J \subset I$ then $\hat{J} \subset I$. Now the reason that we are Cotlarizing $L=v^{1 / 2}\left(H_{3}-\pi_{c^{v}}^{*}\right) M_{u}^{1 / 2}$ instead of just $v^{1 / 2} H_{3} M_{u}^{1 / 2}$ is precisely that it gives us the cancelation

$$
\int v^{1 / 2} L h_{I}=0
$$

for every interval $I$. We now simply use the fact that $H_{3} h_{I}$ is constant on $\hat{J}$ while

$$
\int_{\hat{J}} v^{1 / 2} L h_{J}=-\int_{\hat{J}^{c}} v^{1 / 2} L h_{J}
$$

to write

$$
\begin{aligned}
L_{I J}^{5}= & L_{I J}^{5,1}-L_{I J}^{5,2} \\
= & u_{I}^{1 / 2}\left(\int_{(\hat{J})^{c}} v\left(H_{3} h_{I}\right)\left(H_{3} h_{J}\right)\right) u_{J}^{1 / 2} \\
& -u_{I}^{1 / 2}\left(H_{3} h_{I}\right)\left(x_{J}\right)\left(\int_{(\hat{J})^{c}} v\left(H_{3} h_{J}\right)\right) u_{J}^{1 / 2} .
\end{aligned}
$$


First, we bound $L_{I J}^{5,1}$ by Lemma 2.1. We let

$$
A_{I J}=u_{I}^{1 / 2}\left(\int_{(\hat{J})^{c}} v\left(H_{3} h_{I}\right)\left(H_{3} h_{J}\right)\right)^{1 / 2},
$$

and we let

$$
B_{I J}=\left(\int_{(\hat{J})^{c}} v\left(H_{3} h_{I}\right)\left(H_{3} h_{J}\right)\right)^{1 / 2} u_{J}^{1 / 2} .
$$

We have

$$
\begin{aligned}
\sum_{J \notin B_{j k}} A_{I J} A_{I J}^{*} & \leq u_{I}^{1 / 2}\left(\sum_{J \notin B_{j k}} \int_{(\hat{J})^{c}} \frac{v|I|^{3 / 2}|J|^{3 / 2}}{\rho_{x I}^{2} \rho_{x J}^{2}}\right) u_{I}^{1 / 2} \\
& \leq C u_{I}^{1 / 2}\left(\int \frac{v|I|}{\rho_{x I}^{2}}\right) u_{I}^{1 / 2} \\
& \leq C
\end{aligned}
$$

Here, the penultimate inequality comes from the simple observation that for each $x$,

$$
\sum_{J \in \mathcal{D}_{k}} \frac{1}{\rho_{x J}^{2}} \chi_{\hat{J}^{c}}(x) \leq \frac{C}{|J|^{3 / 2}|I|^{1 / 2}} .
$$

The inequality (viii) is obtained by majorizing the sum by

$$
\frac{1}{|J|} \int_{|J|^{1 / 2}|I|^{1 / 2}}^{\infty} \frac{d x}{x^{2}}
$$

Furthermore,

$$
\begin{aligned}
\sum_{I \in \mathcal{D}_{j}} B_{I J}^{*} B_{I J} & \leq u_{J}^{1 / 2}\left(\sum_{I \in \mathcal{D}_{j}} \int_{(\hat{J})^{c}} \frac{v|I|^{3 / 2}|J|^{3 / 2}}{\rho_{x I}^{2} \rho_{x J}^{2}}\right) u_{J}^{1 / 2} \\
& \leq C\left(\frac{|J|^{1 / 2}}{|I|^{1 / 2}}\right)\left(u_{J}^{1 / 2} \int \frac{|J| v}{\rho_{x J}^{2}}\right) u_{J}^{1 / 2} \\
& \leq C\left(\frac{|J|}{|I|}\right)^{1 / 2}
\end{aligned}
$$

which is the desired estimate for $L_{I J}^{5,1}$. Redefining $A_{I J}$ and $B_{I J}$, we continue by decomposing $L_{I J}^{5,2}=A_{I J} B_{I J}$. First, we let

$$
K_{I J}=\rho_{I J}^{1 /(1+\varepsilon)}\left(H_{3} h_{I}\right)(J),
$$


238 N. H. KATZ AND C. PEREYRA

and we let

$$
A_{I J}=|I|^{1 /(2(1+\varepsilon))} u_{I}^{1 / 2} \rho_{I J}^{-1 /(1+\varepsilon)} K_{I J}^{1 / 2}\left(\int_{(\hat{J})^{c}} v\left(H_{3} h_{J}\right)\right)^{1 / 2}
$$

and

$$
B_{I J}=(|I|)^{-1 /(2(1+\varepsilon))} K_{I J}^{1 / 2}\left(\int_{(\hat{J})^{c}} v\left(H_{3} h_{J}\right)\right)^{1 / 2} u_{J}^{1 / 2} .
$$

We estimate

$$
\begin{aligned}
A_{I J} A_{I J}^{*} \leq & u_{I}^{1 / 2}\left|K_{I J}\right||J|^{3 / 2}\left(|I| \int_{\hat{J}^{c}} \frac{v^{1+\varepsilon}}{\rho_{x J}^{2} \rho_{I J}^{2}}\right)^{1 /(1+\varepsilon)} \\
& \cdot\left(\int_{\hat{J}^{c}} \frac{1}{\rho_{x J}^{2}}\right)^{\varepsilon /(1+\varepsilon)} u_{I}^{1 / 2} \\
\leq & C\left|K_{I J}\right||J|^{3 / 2} u_{I}^{1 / 2}\left(|I| \int_{\hat{J}^{c}} \frac{v^{1+\varepsilon}}{\rho_{x I}^{2}}\right)^{1 /(1+\varepsilon)} \\
& \cdot u_{I}^{1 / 2}\left(\frac{1}{|J|^{1 / 2}|I|^{1 / 2}}\right)^{\varepsilon /(1+\varepsilon)}(|I||J|)^{-1 /(1+\varepsilon)} \\
\leq & C \frac{(|I||J|)^{(1+2 \varepsilon) /(2(1+\varepsilon))}}{\rho_{I J}^{(1+2 \varepsilon) /(1+\varepsilon)}}
\end{aligned}
$$

Here we have used the fact that on $\hat{J}^{c}$, one has $\rho_{I J}^{2} \rho_{x J}^{2} \geq|I||J| \rho_{x I}^{2}$. We sum obtaining

$$
\begin{aligned}
\sum_{J \in \mathcal{D}_{k}} A_{I J} A_{I J}^{*} & \leq \sum_{J \in \mathcal{D}_{k}} \frac{|I|^{(1+2 \varepsilon) /(2(1+\varepsilon))}|J|^{(1+2 \varepsilon) /(2(1+\varepsilon))}}{\rho_{I J}^{(1+2 \varepsilon) /(1+\varepsilon)}} \\
& \leq\left(\frac{|I|}{|J|}\right)^{1 /(2(1+\varepsilon))} .
\end{aligned}
$$

Meanwhile, we compute

$$
\begin{aligned}
B_{I J}^{*} B_{I J} \leq & (|I||J|)^{-(2+\varepsilon) /(2(1+\varepsilon))}|J|^{3 / 2}\left|K_{I J}\right| u_{J}^{1 / 2} \\
& \cdot\left(|J| \int_{\hat{J}^{c}} \frac{v^{1+\varepsilon}}{\rho_{x J}^{2}}\right)^{1 /(1+\varepsilon)} u_{J}^{1 / 2} \\
\leq & C \frac{(|I||J|)^{(1+2 \varepsilon) /(2(1+\varepsilon))}}{\rho_{I J}^{(1+2 \varepsilon) /(1+\varepsilon)}}
\end{aligned}
$$


We conclude that

$$
\begin{aligned}
\sum_{I \in \mathcal{D}_{j}} B_{I J}^{*} B_{I J} & \leq \sum_{I \in \mathcal{D}_{j}} \frac{|I|^{(1+2 \varepsilon) /(2(1+\varepsilon))}|J|^{(1+2 \varepsilon) /(2(1+\varepsilon))}}{\rho_{I J}^{(1+2 \varepsilon) /(1+\varepsilon)}} \\
& \leq\left(\frac{|J|}{|I|}\right)^{(1+2 \varepsilon) /(2(1+\varepsilon))} .
\end{aligned}
$$

which gives the desired estimate on $L_{I J}^{5,2}$.

Finally, we come to $L_{I J}^{6}$. We break up into $L_{I J}^{6}=L_{I J}^{6,1}+L_{I J}^{6,2}$. Here, we let $L_{I J}^{6,1}=L_{I J}^{6}$ when $J \subset I$ and 0 otherwise. As before, we let $D_{I}=u_{I}^{1 / 2} c_{I}^{v}\left(m_{I}(v)\right)^{1 / 2}$. We let

$$
F_{I J}=\left(m_{I}(v)\right)^{-1 / 2}\left(\frac{1}{|I|} \int_{I^{c}} v H_{3} h_{J}\right) u_{J}\left(\frac{1}{|I|} \int_{I^{c}} v H_{3} h_{J}\right)\left(m_{I}(v)\right)^{-1 / 2} .
$$

We have $\left\|D_{I}\right\| \leq C|I|^{1 / 2}$ and we have

$$
F_{I J} \leq \frac{|J|^{3}}{d_{I J}^{4}} u_{I}^{-1 / 2} u_{J} u_{I}^{-1 / 2} .
$$

Recall $d_{I J}$ is the distance from $J$ to the boundary of $I$. Here we are directly applying (3.3). Notice this is the only place where we use it. Since all $J$ 's we are considering are not in $B_{j k}$, we have that $d_{I J} \geq$ $|I|^{1 / 2}|J|^{1 / 2}$, but for most $J$, it is even bigger. We write by definition and cancellation,

$$
-L_{I J}^{6,1}=u_{I}^{1 / 2} c_{I}^{v}\left(\frac{1}{|I|} \int_{I^{c}} v H_{3} h_{J}\right) u_{J}^{1 / 2} .
$$

We let $A_{I J}=L_{I J}^{6,1}$ and $B_{I J}=1$. Then we have

$$
\begin{aligned}
\sum_{\substack{J: J \in \mathcal{D}_{k} \\
J \notin B_{j k}}} A_{I J} A_{I J}^{*} & =\sum_{J} D_{I} F_{I J} D_{I}^{*} \\
& \leq D_{I}\left(\sum_{J \in \mathcal{D}_{k}} \frac{|J|^{3}}{d_{I J}^{4}} u_{I}^{-1 / 2} u_{J} u_{I}^{-1 / 2}\right) D_{I}^{*} \\
& \leq \frac{|J|^{\delta}}{|I|^{1+\delta}} D_{I} D_{I}^{*} \\
& \leq C\left(\frac{|J|}{|I|}\right)^{\delta}
\end{aligned}
$$


for some $\delta>0$ which is the desired estimate on $L_{I J}^{6,1}$, provided we can show that

$$
\sum_{\substack{J: J \in \mathcal{D}_{k} \\ J \notin B_{j k}}} \frac{|J|^{3}}{d_{I J}^{4}} u_{J} \leq \frac{|J|^{\delta}}{|I|^{1+\delta}} u_{I},
$$

where the sum is over $J$ 's contained in $I$ with $d_{I J} \geq|I|^{1 / 2}|J|^{1 / 2}$. We let $\mathcal{Z}_{1}$ be the set of those $J$ 's with $d_{I J} \leq|I|^{3 / 4}|J|^{1 / 4}$ and $\mathcal{Z}_{2}$ be the set of those $J$ 's with $d_{I J} \geq|I|^{3 / 4}|J|^{1 / 4}$. We estimate for $\mathcal{Z}_{1}$, noticing that $\operatorname{card}\left(\mathcal{Z}_{1}\right) \leq(|I| /|J|)^{3 / 4}$,

$$
\begin{aligned}
\sum_{J \in \mathcal{Z}_{1}} \frac{|J|^{3}}{d_{I J}^{4}} u_{J} & \leq \sum_{J \in \mathcal{Z}_{1}} \frac{|J|}{|I|^{2}} u_{J} \\
& \leq\left(\sum_{J \in \mathcal{Z}_{1}} \frac{|J|}{|I|^{2}} u_{J}^{1+\varepsilon}\right)^{1 /(1+\varepsilon)}\left(\sum_{J \in \mathcal{Z}_{1}} \frac{|J|}{|I|^{2}}\right)^{\varepsilon /(1+\varepsilon)} \\
& \leq\left(\frac{1}{|I|}\right) u_{I}\left(\frac{|J|}{|I|}\right)^{\varepsilon /(4(1+\varepsilon))}
\end{aligned}
$$

while for $\mathcal{Z}_{2}$,

$$
\begin{aligned}
\sum_{J \in \mathcal{Z}_{2}} \frac{|J|^{3}}{d_{I J}^{4}} u_{J} & \leq \sum_{J \in \mathcal{Z}_{2}} \frac{|J|^{2}}{|I|^{3}} u_{J} \\
& \leq\left(\frac{|J|}{|I|}\right)\left(\sum_{J \in \mathcal{Z}_{2}} \frac{|J|}{|I|^{2}} u_{J}^{1+\varepsilon}\right)^{1 /(1+\varepsilon)}\left(\sum_{J \in \mathcal{Z}_{2}} \frac{|J|}{|I|^{2}}\right)^{\varepsilon /(1+\varepsilon)} \\
& \leq u_{I}\left(\frac{|J|}{|I|^{2}}\right)
\end{aligned}
$$

This leaves us to bound $L_{I J}^{6,2}$.

By definition, for $I \cap J=\varnothing$,

$$
L_{I J}^{6,2}=D_{I}\left(m_{I}(v)\right)^{-1 / 2}\left(\frac{1}{|I|} \int_{I} v H_{3} h_{J}\right) u_{J}^{1 / 2},
$$

zero otherwise.

We break up

$$
A_{I J}=D_{I}\left(m_{I}(v)\right)^{-1 / 2}\left(\frac{1}{|I|} \int_{I} v H_{3} h_{J}\right)^{1 / 2}
$$


and

$$
B_{I J}=\left(\frac{1}{|I|} \int_{I} v H_{3} h_{J}\right)^{1 / 2} u_{J}^{1 / 2},
$$

and now we obtain bounds easily using the fact that $J \cap I=\varnothing$ and $d_{I J} \geq(|I||J|)^{1 / 2}$. We simply compute

$$
\begin{aligned}
\sum_{\substack{J: J \in \mathcal{D}_{k} \\
J \notin B_{j k}}} A_{I J} A_{I J}^{*} \leq & \left(\sup _{x \in I} \sum_{J \in \mathcal{D}_{k}} \frac{|J|^{3 / 2}}{\rho_{x J}^{2}}\right) D_{I}\left(m_{I}(v)\right)^{-1 / 2} \\
& \cdot\left(\frac{1}{|I|} \int_{I} v\right)\left(m_{I}(v)\right)^{-1 / 2} D_{I}^{*} \\
\leq & C \frac{1}{|I|^{1 / 2}} D_{I} D_{I}^{*} \leq C|I|^{1 / 2}
\end{aligned}
$$

Here we use the fact that

$$
\sum_{\substack{J: J \in \mathcal{D}_{k} \\ J \notin B_{j k} \\ J \cap I=\varnothing}} \frac{|J|^{3 / 2}}{\rho_{x J}^{2}} \leq \frac{C}{|I|^{1 / 2}}
$$

At the same time, seeing that the sum on $I$ merely extends the support of the integral, we obtain

$$
\sum_{I} B_{I J}^{*} B_{I J} \leq \frac{|J|^{1 / 2}}{|I|} u_{J}^{1 / 2}\left(|J| \int \frac{v}{\rho_{x J}^{2}}\right) u_{J}^{1 / 2} \leq C\left(\frac{|J|^{1 / 2}}{|I|}\right) .
$$

Multiplying these two estimates and obtaining decay, we prove Theorem 3.1 .

It may be worth pointing out that if assumptions (3.3) and (3.4) seem unappealing, we can also obtain the same result by assuming a sort of "doubling at infinity" condition for $v$ and $u^{-1}$. Thus it suffices, for example, to assume there is a $\delta>0$ with

$$
\begin{aligned}
& \left(\int_{I^{c}} v H_{3} h_{J}\right)\left(m_{J}(v)\right)^{-1}\left(\int_{I^{c}} v H_{3} h_{J}\right) \\
& \leq\left(\frac{|J|}{d_{I J}}\right)^{\delta}\left(\int_{J^{c}} v H_{3} h_{J}\right)\left(m_{J}(v)\right)^{-1}\left(\int_{J^{c}} v H_{3} h_{J}\right) .
\end{aligned}
$$


Then we simply use

$$
\left\|F_{I J}\right\|=\left\|u_{J}^{1 / 2}\left(\int_{I^{c}} v H_{3} h_{J}\right)\left(m_{J}(v)\right)^{-1}\left(\int_{I^{c}} v H_{3} h_{J}\right) u_{J}^{1 / 2}\right\|
$$

together with the bound on the norm of $D_{J}$ to obtain the same result. This doubling assumption may seem more natural to the reader than the assumption we make, until he realizes that it is not even automatic that this doubling assumption is true for $\delta=0$.

\section{References.}

[CF] Coifman, R. R., Fefferman, C., Weighted Norm inequalities for maximal functions and singular integrals. Studia Math. 51 (1974), 241-250.

[CS1] Cotlar, M., Sadosky, C., On the Helson-Szegö theorem and a related class of modified Toeplitz kernels. Proc. Symp. Pure Math. 35 (1979), 383-407. Amer. Math. Soc.

[CS2] Cotlar, M., Sadosky, C., Majorized Toeplitz forms and weighted inequalities with general norms. In Harmonic Analysis. Lecture Notes in Math. 908 (1982), 139-168. Springer-Verlag.

[D] Duoandikoetxea, J., Análisis de Fourier. Ediciones de la Universidad Autónoma de Madrid, 1991.

[Da] David, G., Wavelets and Singular Integrals on Curves and Surfaces. Springer-Verlag, 1991.

[FKP] Fefferman, R. A., Kenig, C. E., Pipher, J., The theory of weights and the Dirichlet problem for elliptic equations. Ann. of Math. 134 (1991), 65-124.

[F] Fujii, N., A condition for a two-weight norm inequality for singular integral operators. Studia Math. 98 (1991), 175-190.

[G] Gehring, F. W., The $L^{p}$ integrability of the partial derivatives of a quasiconformal mapping. Acta Math. 130 (1973), 265-277.

[HMW] Hunt, R., Muckenhoupt, B., Wheeden, R., Weighted norm inequalities for conjugate function and Hilbert transform. Trans. Amer. Math. Soc. 176 (1973), 227-251.

[HP] Hansen, F., Pedersen, G. K., Jensen's inequality for operators and Löwner's theorem. Math. Ann. 258 (1982), 229-241.

[HS] Helson, H., Szegö, G., A problem in prediction theory. Annali di Mat. Pura ed Applicata 51 (1960), 107-138.

[KP] Katz, N. H., Pereyra, C., Some new results on resolvents of paraproducts. Preprint, 1995. 
[KR] Kadison, R. V., Ringrose, J. R., Fundamentals of the Theory of Operator Algebras. Vol. 1. Academic Press, 1983.

[KR2] Kadison, R. V., Ringrose, J. R., Fundamentals of the Theory of Operator Algebras. Vol. 3. Birkhäuser, 1991.

[M] Meyer, Y., Ondelettes et operateurs II. Hermann, 1990.

[P1] Pereyra, C., Sobolev spaces on Lipschitz curves, Paraproducts, and Related operators. Thesis, Yale University, 1993.

[P2] Pereyra, C., On resolvents of dyadic paraproducts. Revista Mat. Iberoamericana 10 (1994), 627-664.

[TV] Treil, S., Volberg, A., Wavelets and the angle between past and future. J. Funct. Anal. 143 (1997), 269-308.

[TVZ] Treil, S., Volberg, A., Zheng, D., Hilbert Transform, Toeplitz operators and Hankel Operators, and invariant $A_{\infty}$ weights. Preprint, 1996.

Recibido: 23 de octubre de 1.995

Nets Hawk Katz*

Department of Mathematics and Statistics

University of Edinburgh

Edinburgh EH9 3JZ

UNITED KINGDOM

nets@maths .ed.ac.uk

and

Cristina Pereyra

Department of Mathematics and Statistics

University of New Mexico

Alburquerque, NM 87131-1141

U.S.A.

crisp@math.unm.edu

\footnotetext{
* The first author was partially supported by the National Science Foundation Postdoctoral Fellowship.
} 